\title{
Lymph node metastasis from undifferentiated-type mucosal gastric cancer satisfying the expanded criteria for endoscopic resection based on routine histological examination
}

\author{
Toshiaki Hirasawa ${ }^{1}$, Junko Fujisaki ${ }^{1}$, Tetsu Fukunaga ${ }^{2}$, Yorimasa Yamamoto ${ }^{1}$, Toshiharu Yamaguchi ${ }^{3}$, \\ MASAMichi KATORI ${ }^{4}$, and NORIKo YAMAMOTO ${ }^{4}$ \\ ${ }^{1}$ Department of Gastroenterology, Cancer Institute Hospital, Japanese Foundation for Cancer Research, 3-8-31 Ariake, Koto-ku, \\ Tokyo 135-8550, Japan \\ ${ }^{2}$ Department of Gastroenterological and General Surgery, St. Marianna University School of Medicine, Kanagawa, Japan \\ ${ }^{3}$ Department of Surgery, Cancer Institute Hospital, Tokyo, Japan \\ ${ }^{4}$ Department of Pathology, Cancer Institute Hospital, Tokyo, Japan
}

\begin{abstract}
A 58-year-old woman was found to have a 45-mm abdominal mass adjacent to the pancreas on screening ultrasonography, and subsequent esophagogastroduodenoscopy revealed a small gastric cancer $(13 \mathrm{~mm}$ in diameter). We initially performed endoscopic submucosal dissection (ESD), and routine histological examination of the specimen sectioned at $2-\mathbf{m m}$ intervals revealed a poorly differentiated adenocarcinoma and signet ring cell carcinoma confined to the mucosa without lymphatic-vascular capillary involvement or ulceration. These findings satisfied the expanded criteria for ESD we reported recently. We next performed laparoscopic excisional biopsy of the abdominal mass, and histological examination with immunohistochemical staining revealed a metastatic lymph node (LN) resulting from the gastric cancer. Distal gastrectomy with extended lymphadenectomy was then performed and histological examination indicated no residual cancer cells or any additional LN metastases. After the above-described clinical course, further analysis was conducted because of the highly unusual nature of this case; 60 additional deep-cut sections from the resected specimen were performed, with one section showing lymphatic involvement in the mucosa on hematoxylin and eosin staining. This case suggests practical limitations in determining lymphatic involvement through routine histological examination, which may not always be able to detect $\mathrm{LN}$ metastasis.
\end{abstract}

Key words Early gastric cancer - Undifferentiated-type gastric cancer - Lymph node metastasis - Endoscopic mucosal resection $\cdot$ Endoscopic submucosal dissection

\section{Introduction}

The use of endoscopic resection (ER) for the treatment of early gastric cancer (EGC) has become widespread,

Offprint requests to: $\mathrm{T}$. Hirasawa

Received: March 11, 2010 / Accepted: September 30, 2010 primarily because of the successful development of endoscopic submucosal dissection (ESD) [1-3]. ER is minimally invasive and preserves the entire stomach, resulting in a better postoperative quality of life for patients $[4,5]$. ER is indicated for tumors with a negligible risk of lymph node (LN) metastasis [6]. Although the indications for ER specified by the Japanese treatment guidelines are strictly limited to small mucosal tumors with differentiated histology (D-type), expanded indications for some D-type lesions (intramucosal cancers either $\leq 3 \mathrm{~cm}$ in size with no lymphatic-vascular capillary involvement regardless of ulceration findings or with no ulceration regardless of tumor size) have been proposed and accepted by high-volume ER centers $[2,6,7]$.

It remains in dispute, however, as to which undifferentiated-type (UD-type) EGC lesions involve a negligible risk of $\mathrm{LN}$ metastasis. We recently published a study in which 3843 cases of surgically resected UD-type EGCs were examined, with particular emphasis placed on investigating the existence of LN metastasis [8]. Of those cases, 310 tumors satisfied our recently proposed criteria (lesion size $\leq 2 \mathrm{~cm}$ and no ulcerative component or lymphatic-vascular capillary involvement), with none of them having any LN metastasis. The risk of metastasis from such tumors was calculated to be $0-0.96 \%$ and we have proposed this as a new category of expanded criteria for ESD. Much to our surprise, immediately after the publication of this proposal, however, we encountered a patient satisfying all the expanded criteria based on routine histological examination, but who had, nonetheless, LN metastasis.

\section{Case report}

A 58-year-old asymptomatic Japanese woman underwent screening abdominal ultrasonography that 
detected a 45-mm abdominal mass adjacent to the pancreas. She was referred to our hospital for further examination. Blood test results including tumor markers were all within normal limits. Computed tomography (CT) revealed an abdominal mass, $45 \mathrm{~mm}$ in diameter (Fig. 1A), located on the upper border of the pancreas. Positron emission tomography identified a hot uptake in the mass (Fig. 1B), although colonoscopy showed no malignant tumors. Esophagogastroduodenoscopy revealed a slightly depressed lesion, $13 \mathrm{~mm}$ in diameter, on the lesser curvature of the antrum (Fig. 2A,B) and biopsy indicated poorly differentiated adenocarcinoma and signet ring cell carcinoma. We diagnosed this as a T1 lesion and believed that the possibility of LN metastasis was very low.

We subsequently performed ESD on this gastric IIc lesion. The procedure was successfully completed without any complications. Routine histological examination with hematoxylin and eosin (H\&E) staining of the resected specimen, sectioned at 2-mm intervals according to the Japanese classification of gastric carcinoma [9], revealed poorly differentiated adenocarcinoma and signet ring cell carcinoma, $13 \mathrm{~mm}$ in diameter, confined to the mucosa, without lymphaticvascular capillary involvement or ulcerative components. Both the lateral and vertical margins were negative for cancer invasion (Fig. 3A, B), but cancer cells were shown to have invaded the deep mucosa, with some direct contact with the muscularis mucosae (Fig. 3C). According to our latest expanded indications, the procedure we used was regarded as curative ESD. We next performed laparoscopic excisional biopsy of the abdominal mass. It was located in front of the common hepatic artery and looked like an enlarged LN, No. 8a. Histological examination revealed it to be a LN containing poorly differentiated adenocarcinoma. Further immunohistochemical staining strongly suggested that it was a metastasis from the gastric lesion resected by ESD.

A month after the ESD, distal gastrectomy (D2) + paraaortic lymphadenectomy was performed, aiming at complete removal of the possible lymphatic metastasis. Histological examination of the resected stomach showed an ulcer scar after the ESD, without any residual cancer cells. None of the 60 LNs resected contained a metastasis. The postoperative course was uneventful and the patient is currently free of recurrence, on strict surveillance.

This case was so unusual that we decided to conduct additional staining of the 60 deep-cut sections of the original resected specimen with $\mathrm{H} \& \mathrm{E}, \mathrm{D} 2-40$, desmin, Masson, and vimentin for further analysis. Immunohistochemical staining with D2-40 confirmed the lack of any lymphatic-vascular capillary involvement, while immunohistochemical staining with desmin, Masson, and vimentin revealed no evidence of fibrosis in the submucosal layer or deformity of the muscularis mucosae. One of the additional 60 deep-cut sections stained with H\&E, however, showed lymphatic involvement in the deep mucosa (Fig. 3D).

\section{Discussion}

Lymphatic vessels are found in deep gastric mucosa adjacent to and within the muscularis mucosae. The upper and middle levels of the mucosa contain no such lymphatics in normal, inflammatory, hyperplastic, or neoplastic tissue [10-12]. Sako et al. [12] examined the distribution of lymphatic vessels by immunodetection, using D2-40. They reported that lymphatics were densest in the muscularis mucosae and that UD-type gastric cancer without glandular formation may be scattered in the lymphatic vessels. The morphological detection of intralymphatic tumor cells may be more difficult in UD-type than D-type cancers [12]. Based on routine histological examination in the present case, we observed that UD-type cancer cells had invaded the deep mucosa with some direct contact with the muscularis mucosae, but we did not detect any lymphatic involvement. Only one of the additional 60 deep-cut sections stained with $\mathrm{H} \& \mathrm{E}$ revealed lymphatic involvement, so it is possible that the LN metastasis occurred through this route. At first, this case satisfactorily met our expanded criteria for ESD, but a thorough analysis of the additional 60 deep-cut sections clearly established that this case did not satisfy such recently proposed criteria.

According to the Japanese classification of gastric carcinoma [9], endoscopically resected specimens should be serially sectioned at $2-\mathrm{mm}$ intervals. Our case suggests that such routine histological examination, however, may not always be able to detect LN metastasis, especially in those cases involving UD-type gastric cancer which may sometimes be scattered in lymphatic vessels without glandular formation, making it even more difficult to detect the existence of such lymphatic involvement. Cases in which UD-type cancer cells invade the deep gastric mucosa, therefore, should be submitted to deep-cut sectioning and staining, preferably with both $\mathrm{H} \& \mathrm{E}$ and D2-40, but there still are practical limitations in detecting lymphatic involvement. In the present very rare case, a relatively small UD-type mucosal gastric cancer was detected as the direct result of a large LN metastasis first being discovered, but we were only able to detect lymphatic involvement after considerable staining of numerous additional deep-cut sections of the original resected specimen.

We should keep in mind that a tumor with UD-type histology satisfying the expanded criteria does have the possibility, though extremely low (up to $0.96 \%$ ), of 

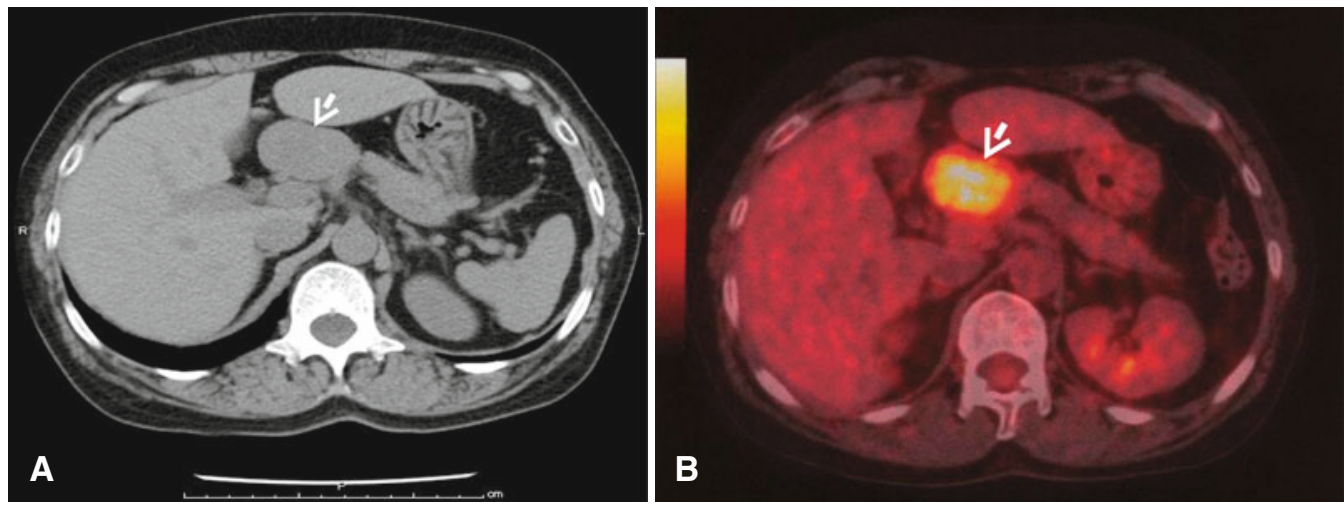

Fig. 1. A Computed tomography revealed a 45-mm tumor (arrow). The patient had a history of bronchial asthma, so contrast medium could not be used. B Positron emission tomography detected a hot uptake in the abdominal mass (arrow)
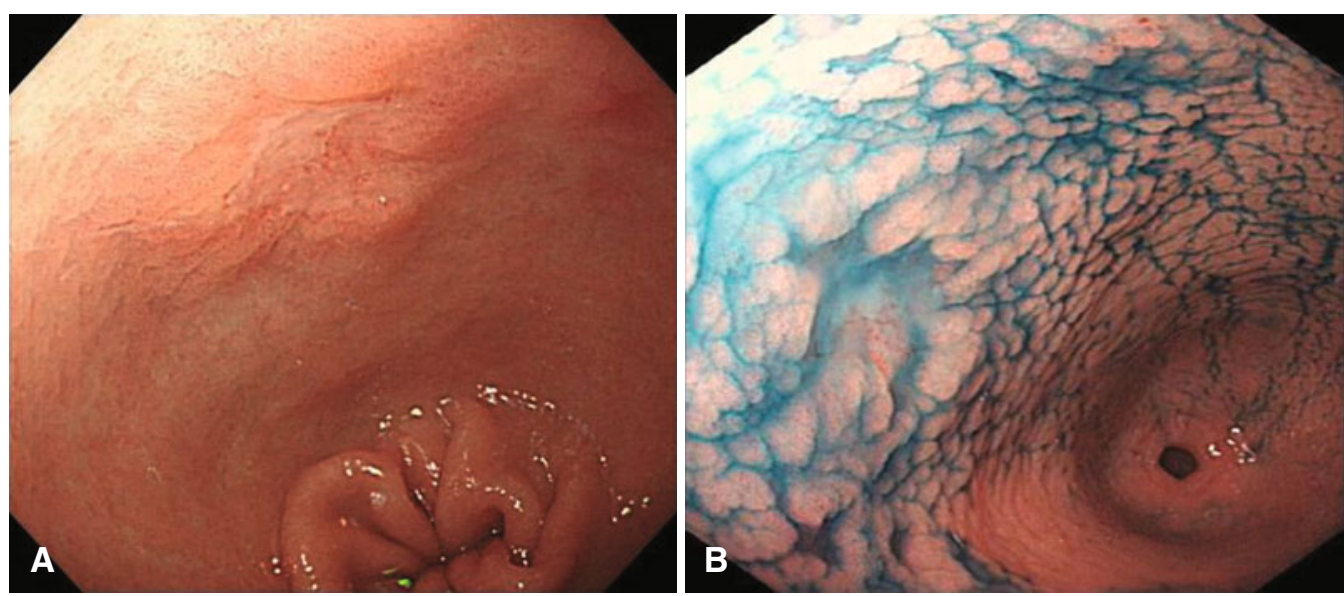

Fig. 2A, B. Esophagogastroduodenoscopy revealed a slightly depressed lesion (0-IIc) on the lesser curvature of the antrum. After indigo-carmine dye spraying, it was demarcated more clearly
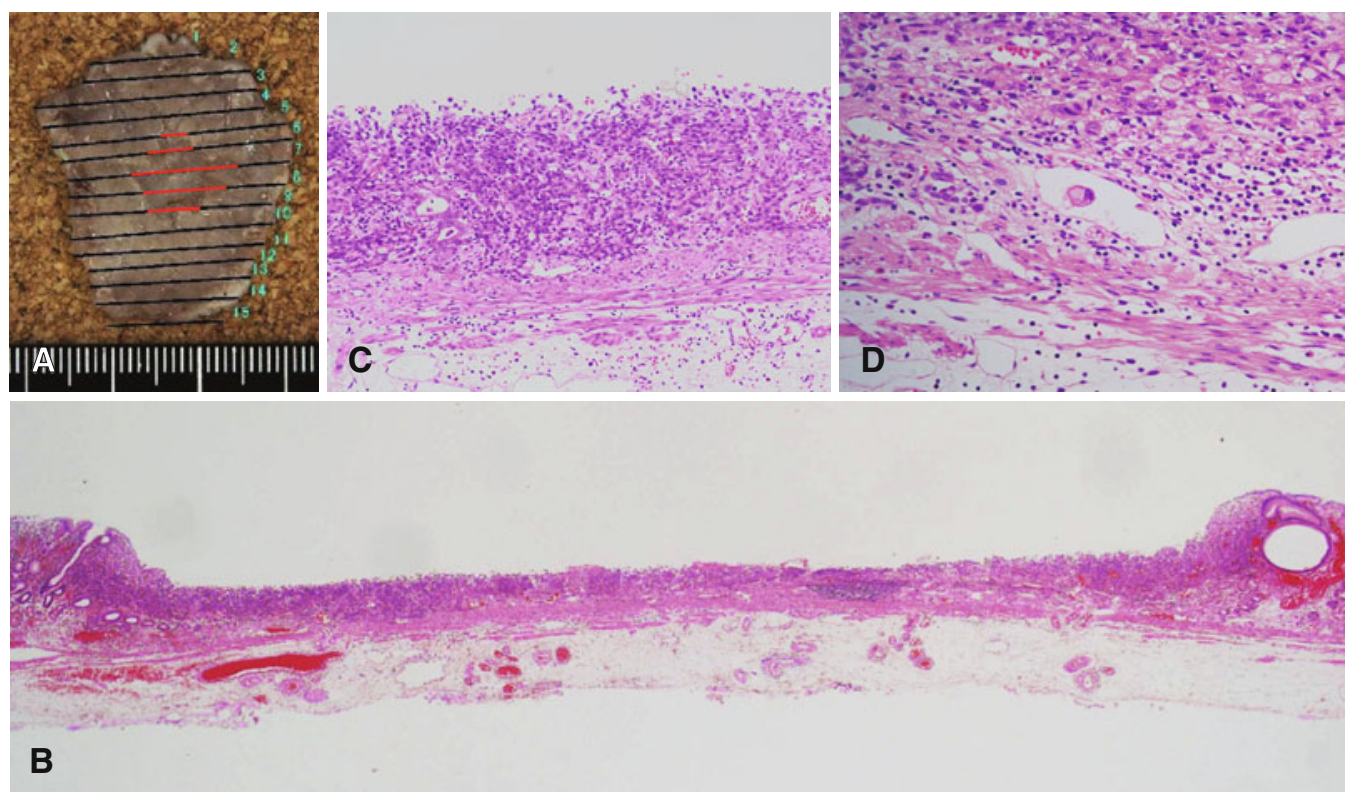

Fig. 3. A Histological mapping of the endoscopic submucosal dissection (ESD) specimen. Red lines indicate tumor measuring $13 \times 8 \mathrm{~mm}$. B Microscopically, the tumor was confined to the mucosa, without ulcerative findings. C Cancer cells invaded the deep mucosa, with some cells in contact with the muscularis mucosae. D Signet ring cell in the lymphatic vessel of a single deep-cut section. B H\&E, ×20; C H\&E, ×200; D H\&E, ×400 
lymph node metastasis, and histological examination has practical limitations. When ER is planned for a UD-type EGC, obtaining detailed informed consent, after disclosure of the slight risk of LN metastasis, is necessary, and careful observation, including CT before and after ER, is essential.

\section{References}

1. Ono H, Kondo H, Gotoda T, Shirao K, Yamaguchi H, Saito D, et al. Endoscopic mucosal resection for treatment of early gastric cancer. Gut 2001;48:225-9.

2. Oda I, Saito D, Tada M, Iishi H, Tanabe S, Oyama T, et al. A multicenter retrospective study of endoscopic resection for early gastric cancer. Gastric Cancer 2006;9:262-70.

3. Chung IK, Lee JH, Lee SH, Kim SJ, Cho JY, Cho WY, et al. Therapeutic outcomes in 1000 cases of endoscopic submucosal dissection for early gastric neoplasms: Korean ESD Study Group multicenter study. Gastrointest Endosc 2009;69:1228-35.

4. Davies J, Johnston D, Sue-Ling H, Young S, May J, Griffith J, et al. Total or subtotal gastrectomy for gastric carcinoma? A study of quality of life. World J Surg 1998;22:1048-55.
5. Nunobe S, Sasako M, Saka M, Fukagawa T, Katai H, Sano T. Symptom evaluation of long-term postoperative outcomes after pylorus-preserving gastrectomy for early gastric cancer. Gastric Cancer 2007;10:167-72.

6. Gotoda T. Endoscopic resection of early gastric cancer. Gastric Cancer 2007;10:1-11.

7. Gotoda T, Yanagisawa A, Sasako M, Ono H, Nakanishi Y, Shimoda $\mathrm{T}$, et al. Incidence of lymph node metastasis from early gastric cancer: estimation with a large number of cases at two large centers. Gastric Cancer 2000;3:219-25.

8. Hirasawa T, Gotoda T, Miyata S, Kato Y, Shimoda T, Taniguchi H, et al. Incidence of lymph node metastasis and the feasibility of endoscopic resection for undifferentiated-type early gastric cancer. Gastric Cancer 2009;12:148-52.

9. Japanese Gastric Cancer Association. Japanese classification of gastric carcinoma - 2nd English edition - Gastric Cancer 1998;1:10-24.

10. Lehnert T, Erlandson RA, Decosse JJ. Lymph and blood capillaries of the human gastric mucosa. A morphologic basis for metastasis in early gastric carcinoma. Gastroenterology 1985;89:939-50.

11. Listrom MB, Fenoglio-Preiser CM. Lymphatic distribution of the stomach in normal, inflammatory, hyperplastic, and neoplastic tissue. Gastroenterology 1987;93:506-14.

12. Sako A, Kitayama J, Ishikawa M, Yamashita H, Nagawa H. Impact of immunohistochemically identified lymphatic invasion on nodal metastasis in early gastric cancer. Gastric Cancer 2006;9:295-302. 